\title{
DISTRIBUCION ESPACIAL Y TEMPORAL DE ACANTHODACTYLUS ERYTHRURUS (SAURIA: LACERTIDAE) EN UN ARENAL COSTERO ALICANTINO .
}

\author{
Por E.SEVA ${ }^{1}$ y A.ESCARRE ${ }^{1}$
}

\section{INTRODUCCION}

En el estudio de la ecología de los reptiles es ya casi tradicional considerar alimento, hábitat y tiem po como las tres dimensiones principales que configuran los distintos tipos de aprovechamiento de recursos (Pian $\mathrm{ka}, 1969$; Schoener, 1974) tanto a nivel interespecífico como intraespecífico. De ellos, la dimensiôn temporal ha sido sin embargo la que menos atención ha recibido (Simon y Middendorf, 1976).

En una gran mayoría de los estudios que se lle van a cabo para definir el microhabitat de las especies, se hace especial hincapié en los aspectos diferenciales de manera tal que es como si se procediese a muestrear del espacio, únicamente aquellos puntos en que se obser van especies. De esta forma se pueden evaluar mejor las preferencias relativas de las distintas especies o cla ses ontogénicas, que la selección de características dē espacio total que cada reptil lleva a cabo en mayor ome nor proporción.

La heterogeneidad en los diversos componentes del espacio condiciona el modo de distribución de las es pecies que 1o ocupan, siendo frecuente que la densidad

1. Departamento de Biología . Facultad de Ciencias . Universidad de Alicante. 
de individuos varie considerablemente de unos lugares a otros, variación que como apuntan Fleming y Hooker(1975) viene de la mano de numerosos aspectos intrínsecos y ex trínsecos complicadamente relacionados, por lo que la medida de ciertas variables aisladas sólo da una corta perspectiva de dichas relaciones.

Los cambios de la explotación de recursos en el tiempo son una de las respuestas de la especie a las va riaciones cíclicas temporales a que están sometidas cier tas variables que tienen como respuesta la distribución cíclica de la actividad de la especie.

En el presente trabajo se incluyen algunos de los resultados preliminares sobre un estudio más amplio que abarca toda la taxocenosis de reptiles de un arenal costero que tiene como especie dominante el Acanthodactyzus emythmus, que pertenece a un género sobre el que existen escasos estudios de tipo ecológico (Valverde, 1967 ; Busack, 1976; Pough y Busack, 1978; Cisse, Karns y Karns, 1977; y Bons, 1972 .

El objeto principal del estudio es la descripción de la distribución espacio-temporal de la especie, $y$ de las diferencias existentes entre sexos y clases de edad. En el aspecto espacial hay que resaltar que en la delimitación de los microhabitats se ha partido de una información completa e independiente de las observaciones sobre tres características, una del biotopo, la pen diente, y dos de la biocenosis, la cobertura y tipo de vegetación. Estas últimas son un buen reflejo de otros caracteres físicos : la granulometría y compacidad del substrato. En la dimensión temporal se observan tanto los cambios diarios de actividad como los estacionales.

\section{ZONA DE ESTUDIO}

En la mitad sur de la provincia de Alicante se localizan ciertos acúmulos arenosos que conforman fran jas de dunas costeras intermitentes de una anchura que varía entre 300 y $500 \mathrm{~m}$. De los tres arenales originales (San Juan, Saladar y Guardamar), el primero ha sido fuer 


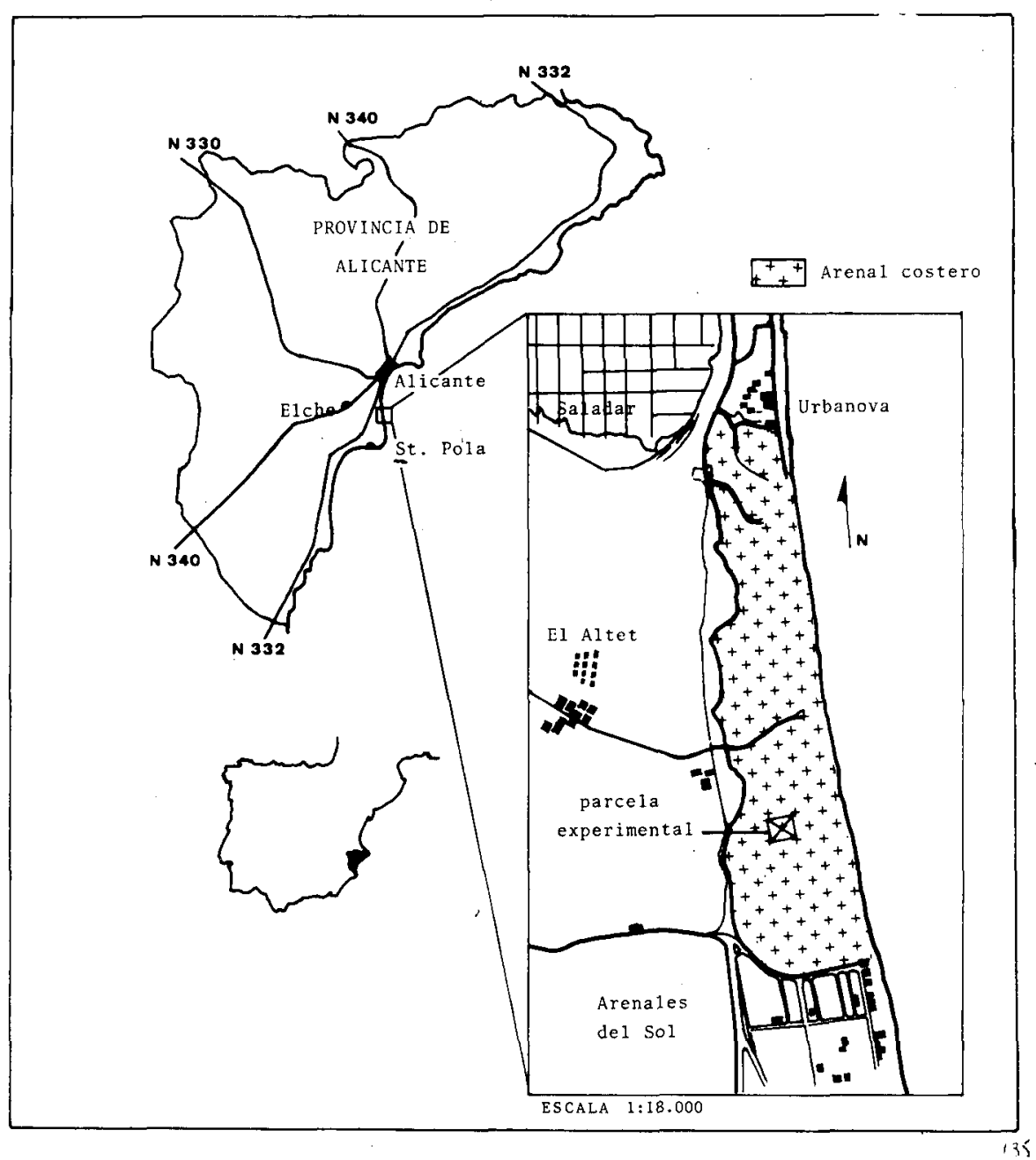

Figura 1 . Localización geográfica de la parcela experimental Geographical location of the experimental plot 
temente impactado por la urbanización llevada a cabo en los ultimos veinte años, mientras que el tercero ha sido fijado con una repoblación de Pinus pinea que data de principios de siglo . El arenal de la playa del Saladar no ha escapado por completo a la demanda turística de es pacios costeros, pero ha quedado una zona comprendida en tre las urbanizaciones Urbanova y Arenales del Sol, de una extensión aproximada de $100 \mathrm{Ha}$., que se ha mantenido relativamente intacta, y ha conservado mejor la flora y fauna (Sauleda, comunicación verbal) propia de este bio topo.

La parcela experimental es un rectángulo de $100 \mathrm{~m}$ por $80 \mathrm{~m}$. y está situada en el centro del arenal de la playa del Saladar (figura 1). Las coordenadas U.T.M.de uno de sus vértices son YH168384.

E1 substrato de la parcela está formado en parte por areniscas compactadas, que constituyen el terreno más duro de la zona y ocupan un $40 \%$ de la superficie, por porciones de duna móvil de gran pendiente y que cu bren alrededor de1 $10 \%$, por una porción central más $1 \bar{i}$ mosa (5\%) y por ultimo las dunas fijadas de menor relieve y representan el $45 \%$ restante.

\section{METODOLOGIA}

La parcela de estudio se ha dividido en 80 cua drados de $100 \mathrm{~m}^{2}$, cada uno de 10 s cuales se subdividió a su vez en 8 triángulos iguales de $12.5 \mathrm{~m}^{2}$; como referen cia se utilizaron botes enterrados en el suelo con algún elemento conspicuo al exterior que permitía la fácil lo calización. Los 640 pequeños triángulos de la parcelá se han tomado como las minimas unidades de espacio, a las que no se asigna más que un cierto tipo de vegetaciỏn, pendiente o cobertura vegetal y en el interior de las cuales no se situan más detalladamente las observaciones de lagartijas.

Se ha realizado un levantamiento topozráfico de 1a parcela de estudio a escala 1:100 con curvas de mivel 
para equidistancia de cota de $0.25 \mathrm{~m}$. En cada uno de 10 s triángulos se han contabilizado el número de curvas de nivel presentes como una evaluación de la pendiente.

Para el estudio de la vegetación se han hecho inventarios florísticos de cada uno de los triângulos. De ellos se hace constar 1a cifra de cobertura total del área por la vegetación, y la lista de las especies pre sentes para las que se estima la cobertura según la mis ma escala de tres valores, usada para la cobertura total y cuya correspondencia es la siguiente : 1 , cobertura $\underline{i}$ gual o inferior al $10 \%$ de la superficie; 2 , cobertura comprendida entre el 10 y el $30 \%$ de la superficie y 3 , cobertura igual o superior al $30 \%$.

La tipificación de la vegetación se inició con una ordenación de los inventarios por medio del análisis factorial de correspondencias con la versión (CRSP) que el Centro de Proceso de Datos del Ministerio de Educación y Ciencia ofrece a la Red de Usuarios Externos en el RUExpres de Enero de 1979 . Debido a la dimensión del problema se procesaron separadamente los 160 inventarios de cada una de las cuatro filas de cuadrados de $20 \times 20 \mathrm{~m}$. y un número de especies que varía entre 43 y 48 . Las fi las de cuadrados de este tamaño se eligieron para evitar que algún tipo de vegetación fuese exclusivo de una de ellas y además, por su orientación, se pretendía que a barcasen el máximo de heterogeneidad de la parcela. Eñ las cuatro representaciones obtenidas del análisis de co rrespondencias, se han encontrado grupos de especies que se repiten en dos o más ánálisis y de ellas, se han ele gido las más próximas a los centros de gravedad de los grupos de inventarios que forman los distintos tipos de vegetación. Con este procedimiento existen 34 inventarios que no resultan fáciimence asignables a un único ti po de vegetación.

En Ios años 1977 y 1978 los ejemplares, captura dos a mano, se marcaban con una combinación de cortes de dedos y se les señalaba el dorso con marcas coloreadas que permitian la identificación de individuos sin nece sidad de aprehenderlos. En las temporadas de 1979 y 1980 se ha prescindido de las marcas de pintura y, por exigencias de otros registros (temperaturas, cloacales, peso...), se han capturado todos los ejemplares. 
Como norma, se tratan como juveniles los machos que no sobrepasan los $52 \mathrm{~mm}$. de longitud de cabeza y cuerpo (LCC) y aquellas hembras que no superan 1 os $50 \mathrm{~mm}$. de LCC . Busack (1976) considera juveniles de ambos sexos a aquellos ejemplares con una LCC inferior a $60 \mathrm{~mm}$, pero existen datos de individuos sexualmente maduros y que no alcanzan dicha longitud.

Para conocer los ciclos de actividad diaria a lo largo de los distintos meses del año, se ha recurrido a un transecto fijo de poco más de $1 \mathrm{Km}$. de largo y una amplitud de perturbación de $5 \mathrm{~m}$. Los ejemplares no se han capturado, lo que ha dado lugar a que, en ocasiones, el reconocimiento del sexo en individuos adultos haya si do incierto. El trayecto completo elegido, no interfiere con la parcela original de estudio,y fue trazado de ma nera que atravesase las zonas más representativas de la tipología de la vegetación.

El tiempo empleado ha sido el de una jornada com pleta de cada mes, recorriendo el itinerario periódicamente cada hora a partir de las 5.00 solares con el in dicativo de final de actividad, de un recorrido completo sin avistaniento de ejemplares. Los días elegidos han sido soleados o con minima presencia de nubes ya que, en meses templados, 1igeras interrupciones de radiación di recta se ven traducidas en un descenso patente de la ac tividad (Heatwole, 1976; Avery, 1978).

\section{RESULTADOS}

\section{TOPOGRAFIA, COBERTURA Y TIPOS DE VEGETACION}

En la figura 2 viene representado el mapa de pendientes de la parcela de estudio. Los triángulos que dan clasificados arbitrariamente en tres tipos: aquellos que no contienen ninguna curva de nivel, los que representan una o dos (desnivel máximo de $0.5 \mathrm{~m}$.) y los que contienen tres o más curvas. Las zonas más llanas ocu pan una pequeña extensión en el centro del rectángulo y un reducido número de triángulos dispersos. Las zonas 


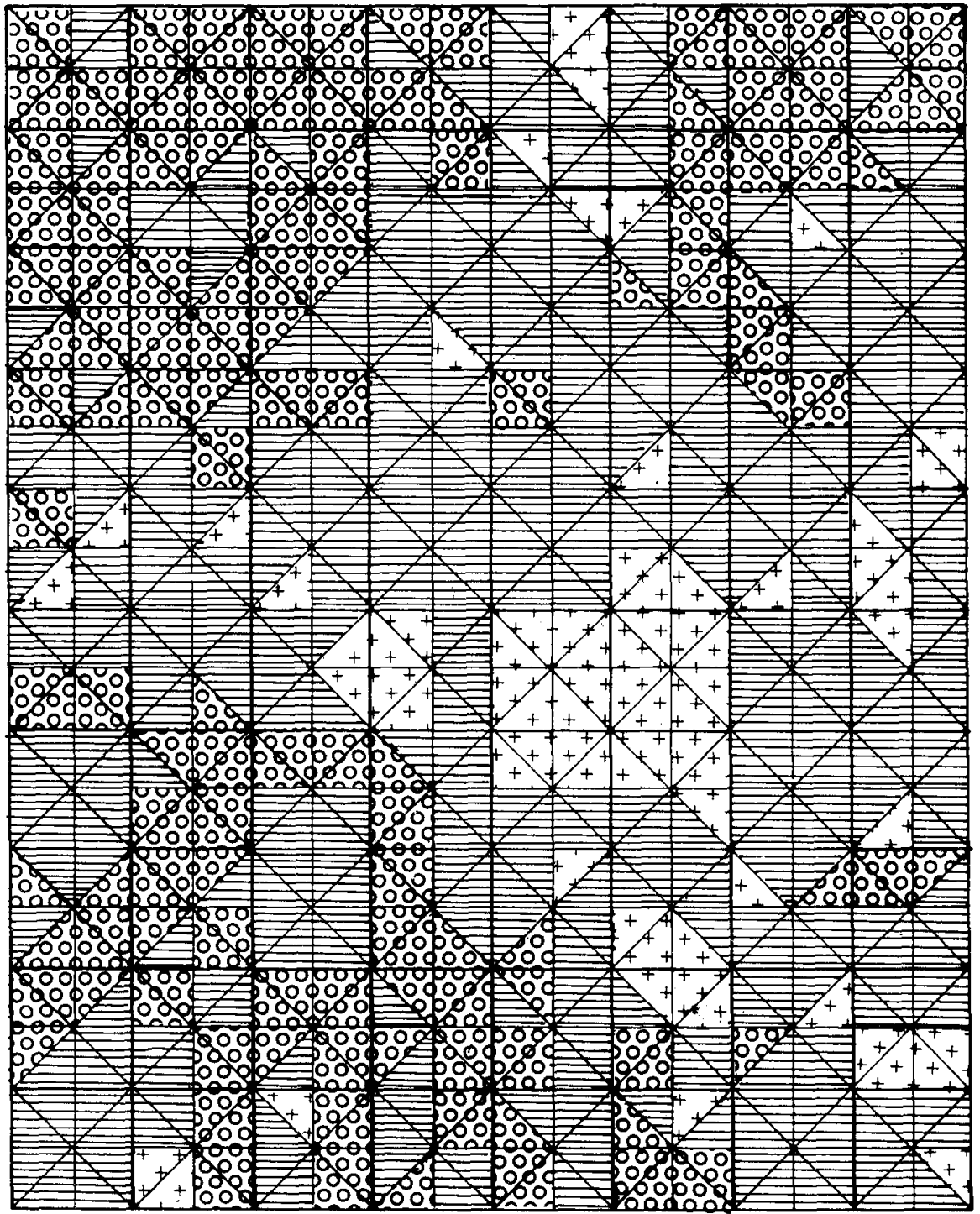

$[++10$ curvas nivel pood 3 ó más curvas

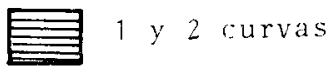

Figura 2 . Mapa de pendientes de 1 a parcela experimental, por uni dades elementales de superficie, según las clases está blecidas en el capitulo 3 .

Slope map of the experimental plot by elemental surface units (see chapter 3 ). 

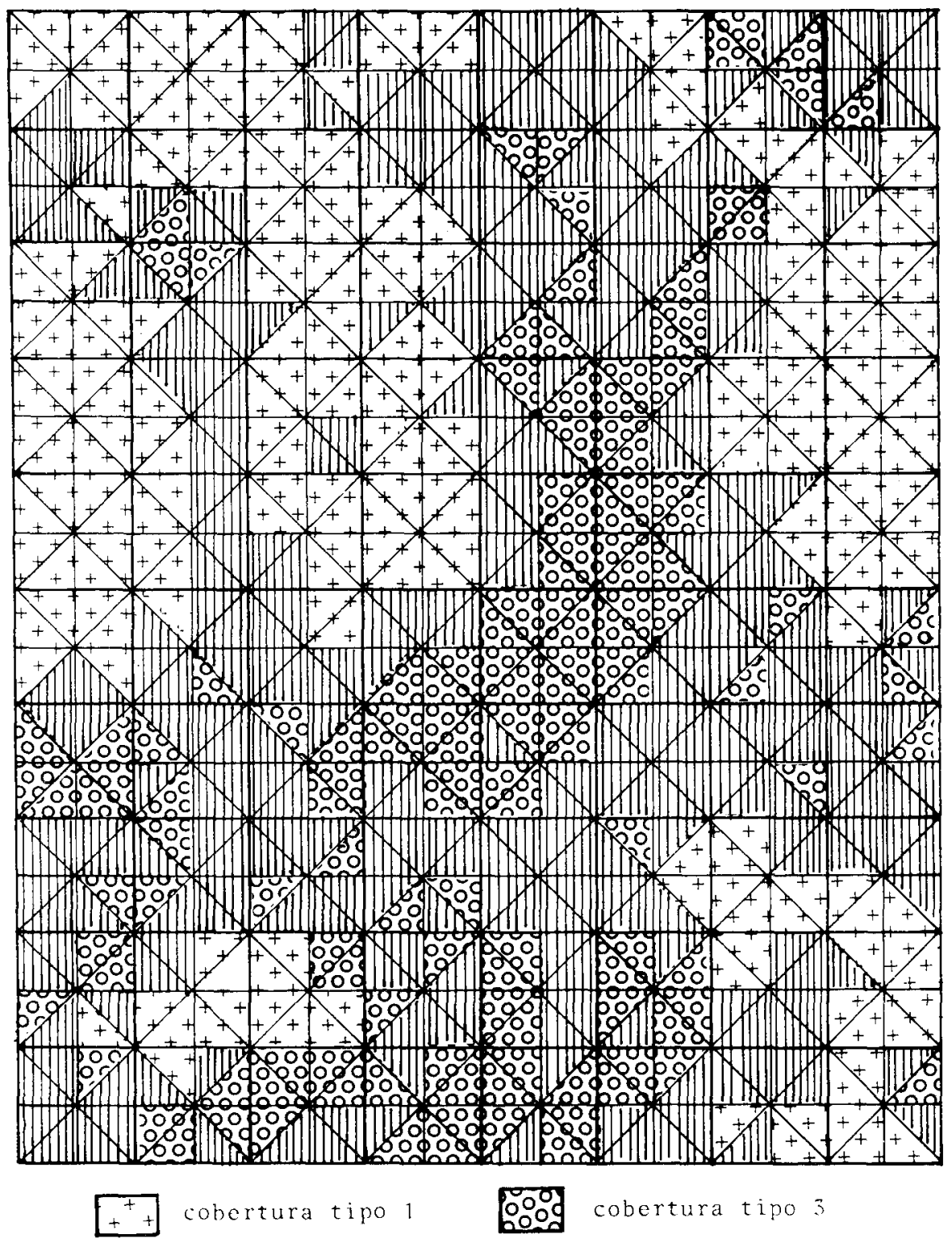

IIIII cobertura tipo 2

Figura 3. Mapa de cobertura dada por la vegetación en unidades bâsicas de superficie.

Map of vegetation cover in the elemental surface units. 
de mayor relieve se concentran en los dos vértices supe riores $\mathrm{y}$ en un área bastante irregular cercana al vérti ce inferior izquierdo.

E1 mapa de cobertura dada por la vegetación se ha representado en la figura 3 , de acuerdo al criterio expuesto en el capítulo de metodología. La zona más cu bierta comprende una franja que atraviesa longitudinalmente la cuadrícula y un ensanche que ocupa gran parte del cuadrante inferior izquierdo. La superficie de me nor cobertura se identifica con las zonas de gran pendiente que rodean a los dos vértices superiores.

En la clasificación de la vegetación, se han ob tenido cuatro tipos, cuyas especies más representativas figuran en la tabla 1 , y que tiene una buena correspondencia con la textura y compacidad del substrato. El ma pa que representa la distribución de los distintos tipos de vegetación, corresponde al de la figura 4 .

\section{DISTRIBUCION ESPACIAL}

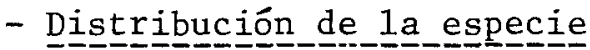

Las observaciones de la distribución espacial de la lagartija colirroja están resumidas en la tabla 2. Para cada uno de los triángulos, que representan la minima unidad de espacio establecida, se han considerado únicamente tres factores: una del biotopo, la pendiente, y dos de la biocenosis, la cobertura y el tipo de vegetación. Para la construcción de dicha tabla se han cal culado los valores teóricos de las observaciones del ani mal, haciéndolos proporcionales al porcentaje de cada tí po de pendiente, ${ }_{2}$ cobertura o tipo de comunidad vegetal. Los valores de $X^{2}$ manifiestan la selección de determina das clases de estos atributos por 1a especie.

En relación con la pendiente, es manifiesta la preferencia por zonas llanas, poca diferencia en pendien tes medias, y sin embargo, poca tendencia a habitar $1 \bar{u}$ gares de gran pendiente. 


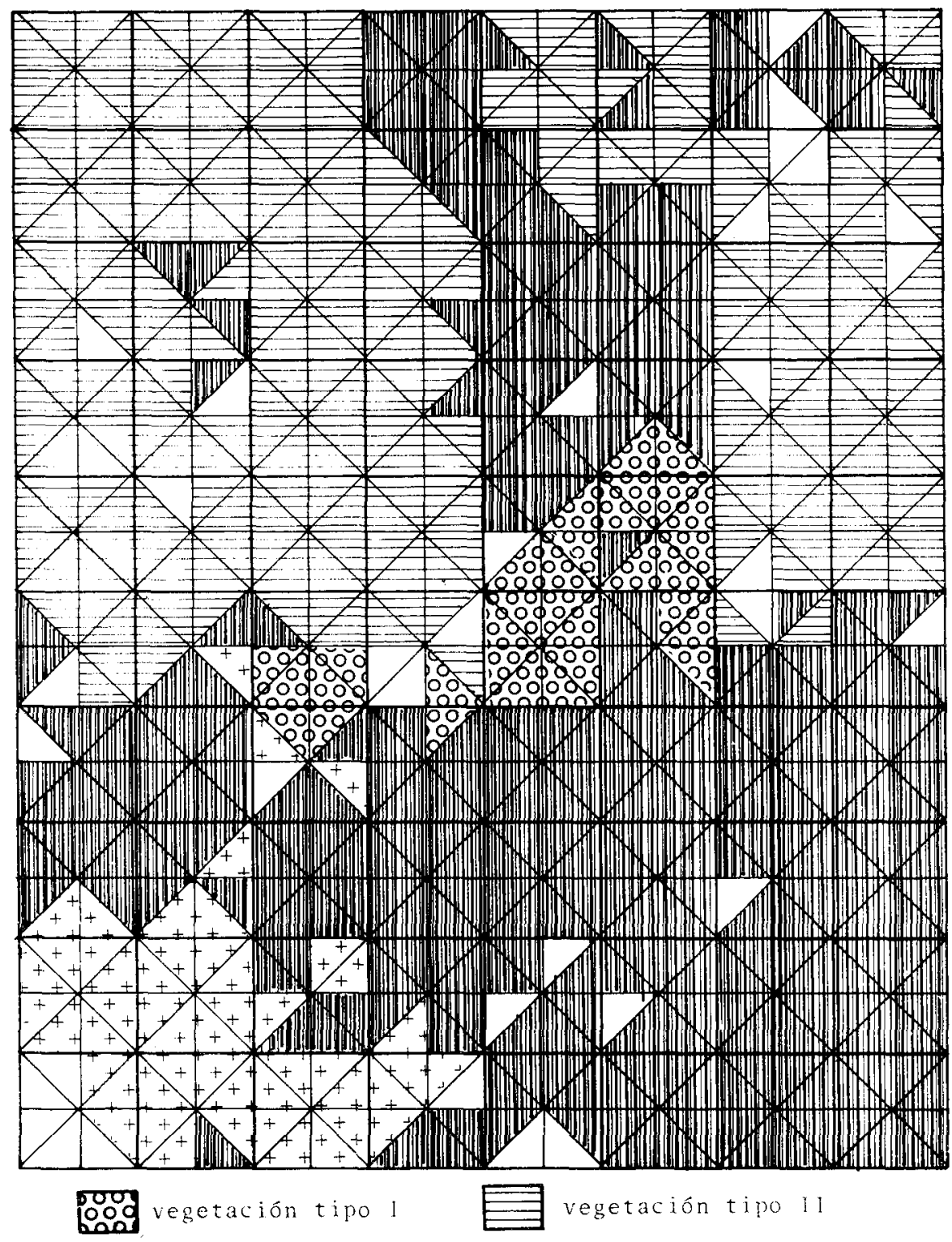

III vegetación tipo II $+_{+}^{+}$vegetación tipo IV

Figura 4 . Mapa de distribución de los distintos tipos de vegetación en la parcela. Los triángulos que figuran en blan co no son asignables a un único tipo.

Distribution map of the different types of vegetation in the study plot. White areas aren't assigned to a single type. 


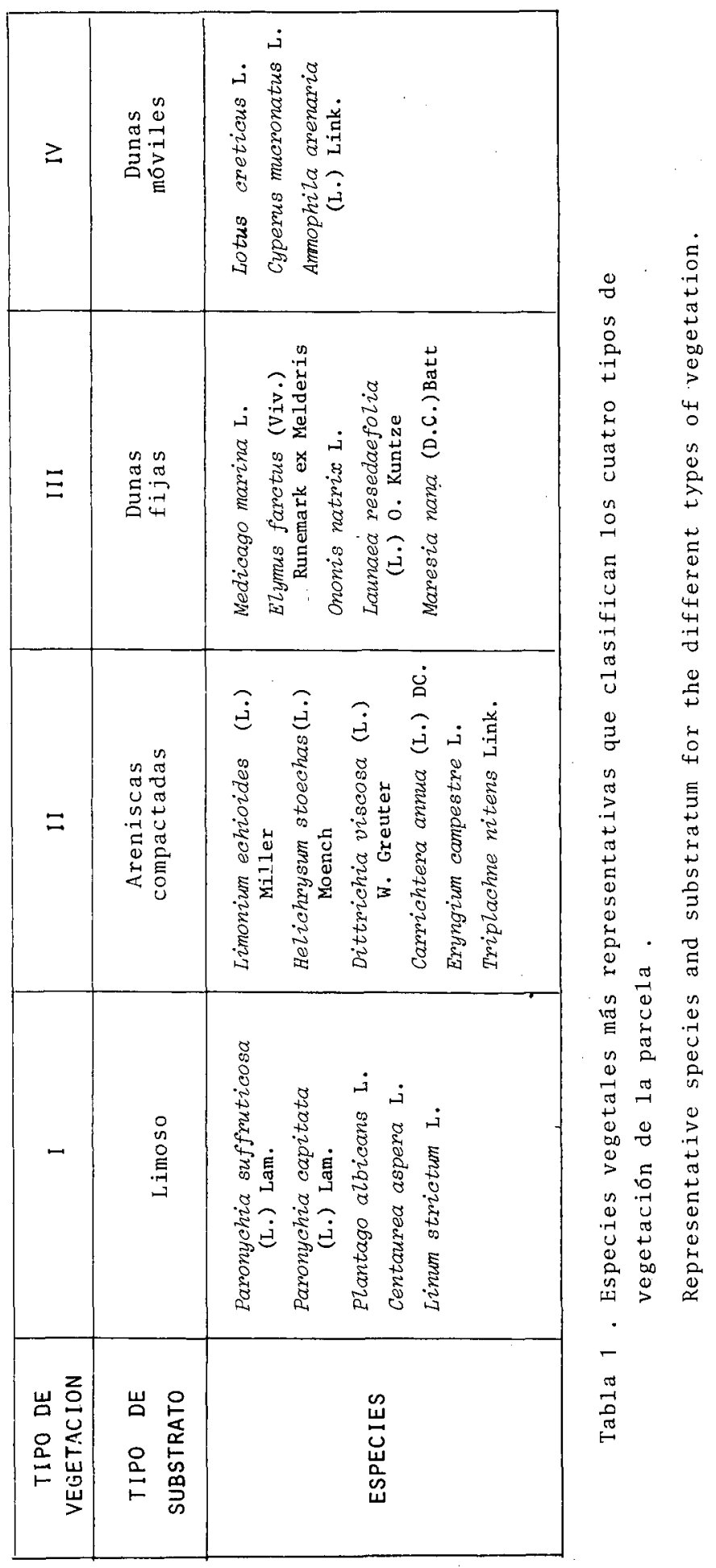


Tabla 2 . Número de capturas de Acanthodactylus erythru mus para cada clase de pendiente, cobertura vegetal y tipo de vegetación. Se dan también los valores teóricos que corresponderían si la especie no discriminase entre las características reseñadas y los valores y nivel de significación de la $x^{2}$.

Capture number of A.erythrurus for each class of slope, covering and vegetation type. The table also shows theoretic values as if the species didn't discriminate between the consi dered characteristics. The values and signification level of $x^{2}$ also appear.

\section{$\mathrm{N}^{\circ} \mathrm{DE}$ CURVAS DE NIVEL}

POR TRIANGULO

PENDIENTE

Observado

Esperado

COBERTURA DE VEGETACION

Observado

Esperado

TIPOS DE VEGETACION

Observado

$\begin{array}{llll}57 & 107 & 446 & 55\end{array}$

Esperado

$36.3 \quad 258.7 \quad 316$

54

$\chi^{2}=154.3$

$(\mathrm{p}<0.001)$ 
Más manifiesta todavía es la segregación de $A$. erythmurus respecto a las clases de cobertura vegetal : bajo número de observaciones en zonas escasamente cubier tas, y más frecuentes de las esperadas en valores medios y sobre todo altos de la cubierta vegetal.

Respecto a los tipos de vegetación, se observa una clara preferencia hacia el tipo III y en parte hacia el tipo I, a costa únicamente del tipo II, ya que para el tipo IV no existe apenas discrepancia entre los valo res observados y los esperados.

\section{- Diferencias en 1a distribución espacial por sexos y clases de edad.}

Las tablas 3, 4 y 5 expresan los valores de ob servaciones o capturas de $A$. erythmus para cada uno de los tres factores considerados anteriormente, distin guiendo el sexo y la clase de edad según la norma indi cada en la metodología.

En el factor pendiente se evidencia un distinto comportamiento de los sexos; los machos no son afectados en su distribución y las hembras, más las adultas que las juveniles, muestran preferencia por zonas de escaso relieve.

Respecto a la cobertura vegetal, ambos sexos y clases de edad muestran gran preferencia por las zonas más cubiertas. Individuos juveniles machos muestran ten dencia específica por zonas de cobertura $3\left(x^{2}=20.2 \overline{5}\right.$ para la selección entre 2 y 3 ), mientras que machos adultos muestran preferencia por coberturas 2 y 3 indis tintamente, tendencia compartida por hembras juvrniles. Las preferencias de hembras adultas son por coberturas superiores al 30\% ( $x^{2}=12.19$ para la selección entre 2 y 3 ).

Para los diferentes tipos de vegetación, se ob servan comportamientos poco generalizables por edades 0 sexos . El tipo I, indiferente para juveniles, es ligeramente preferido por machos adultos, y obtiene la máxi ma aceptación por parte de las hembras adultas. El tipo II es rechazado en todos los casos en parecida proporción. En zonas de vegetación tipo III se ha observado 
Tabla 3 . Número de capturas de Acanthodactylus erythru rus distribuidas por sexos y tamaños, para ca da clase de pendiente. (Ver leyenda tabla 2) Capture number of Acanthodactylus exythrurus distributed by sex and size, for each class of slope.

\begin{tabular}{l} 
PENDIENTE \\
$\left(\mathrm{N}^{\circ} \mathrm{DE}\right.$ CURVAS POR TRIANGULO $)$ \\
$0 \quad 1$ y $2 \quad 3$ ó más \\
\hline
\end{tabular}

Juvenil M

Observado

25

117

53

Esperado

22.2

111.3

$61.5 \quad x^{2}=1.8$

Juvenil $\mathrm{H}$

Observado

38

127

58

Esperado

25.4

127.2

$70.4 \quad \chi^{2}=8.3$

$(\mathrm{p}<0.05)$

Adulto M

$\begin{array}{lllll}\text { Observado } & 25 & 95 & 44 & \\ \text { Esperado } & 18.7 & 93.5 & 51.8 & \chi^{2}=3.3\end{array}$

Adulto $\mathrm{H}$

Observado

22

66

22

Esperado

12.5

62.8

$34.7 \quad \chi^{2}=11.9$

$(\mathrm{p}<0.01)$ 
Tabla 4. Número de capturas de Acanthodactylus erythru mus distribuidas por sexos y tamaños, para cá da clase de cobertura de vegetación. (Ver le yenda tabla 2).

Capture number of A.erythrurus by sex and si ze for each class of vegetation cover (see le gend table 2).

\section{COBERTURA DE VEGETACION}

\begin{tabular}{llll}
1 & 2 & 3 \\
\hline & & & \\
23 & 89 & 82 & \\
66.7 & 86.7 & 40.6 & $x^{2}=\underset{(p<0.001)}{72.57}$
\end{tabular}

Juvenil $\mathrm{H}$

Observado

33

123

70

Esperado

$77.7 \quad 101$

47.3

$$
\begin{aligned}
& \chi^{2}= 41.39 \\
&(p<0.001)
\end{aligned}
$$

Adulto $M$

$\begin{array}{lllll}\text { Observado } & 13 & 95 & 61 \\ \text { Esperado } & 58.1 & 75.5 & 35.4 & \chi^{2}=\begin{array}{l}58.6 \\ (p<0.001)\end{array}\end{array}$

Adulto $\mathrm{H}$

Observado

Esperado

$\begin{array}{cc}9 & 50 \\ 36.4 & 47.4\end{array}$

22.2

$$
\begin{aligned}
& x^{2}= \\
& \quad(p<0.5 \\
& (p<001)
\end{aligned}
$$


Tabla 5. Número de capturas de Acanthodactylus erythru mis distribuidas por sexos y tamaños, para ca da tipo de vegetación. (Ver leyenda tabla 2). Capture number of A.erythrumus by sex and size for each vegetation type. (See legend table 2).

TIPO DE VEGETACION

I II III IV

Juvenil M

$\begin{array}{llllll}\text { Observado } & 12 & 33 & 117 & 21 & \\ \text { Esperado } & 10 & 71.2 & 87 & 14.8 & \chi^{2}=33.8 \\ & & & & & (p<0.001)\end{array}$

Juvenil $\mathrm{H}$

$\begin{array}{llllcc}\text { Observado } & 13 & 43 & 157 & 6 & \\ \text { Esperado } & 12 & 85.2 & 104 & 17.8 & \chi^{2}=55.7 \\ & & & & & (p<0.001)\end{array}$

Adu1to $M$

$\begin{array}{llllll}\text { Observado } & 13 & 21 & 109 & 18 & \\ \text { Esperado } & 8.8 & 62.7 & 76.5 & 13 & \begin{array}{c}\chi^{2}=45.3 \\ (p<0.001)\end{array}\end{array}$

Adul to $\mathrm{H}$

$\begin{array}{lrllll}\text { Observado } & 19 & 10 & 63 & 10 & \\ \text { Esperado } & 5.6 & 39.7 & 48.4 & 8.3 & \begin{array}{l}\chi^{2}=59.4 \\ (p<0.001)\end{array}\end{array}$


superabundancia de elementos juveniles hembras y, en me nor grado, machos adultos, machos juveniles y hembras a dultas. Por último, el tipo IV es casi indiferente pa ra todos los casos, excepto para individuos juveniles hembras, que lo segregan claramente.

\section{DISTRIBUCION TEMPORAL}

Los datos obtenidos de la actividad en los dis tintos meses, figuran en la tabla 6 , que muestra el nûu mero de ejemplares avistados en el trayecto, separados por sexos y tamaños.

Para cada uno de los días muestreados, se han extraido los datos de las temperaturas ambientes que co rresponden a las $7.00 \mathrm{~h} ., 13.00 \mathrm{~h}$. y 18.00 horas, asi co mo la temperatura máxima de la jornada, procedentes del Observatorio Meteorológico del Aeropuerto de Alicante, distante $2 \mathrm{Km}$. del área de estudio, y que figuran en la misma tabla.

Hemos representado en la figura 5 los histogra mas de actividad para sexos y clases de edad, dados por el número de ejemplares vistos en cada hora. Los presu puestos básicos obtenidos a partir de estos resultados, pueden resumirse en los siguientes puntos que posterior mente pasaremos a discutir :

1. En aquellos meses en que existe actividad de individuos juveniles como tales, éstos emergen del refugio con anterioridad a los adultos, excepto en Abril y Julio.

2. La curva de actividad diaria para adultos y ju veniles es unimodal en los meses templados de primavera $\mathrm{y}$ otoño.

3. En meses más calurosos (Mayo, Junio, Julio y Agos to), la curva de actividad es manifiestamente bimodal, con un primer pico de actividad matinal mayor que el se gundo. Coincide con los meses de máximo rango diario de actividad.

4. En los meses más fríos (Noviembre,Diciembre, Ene ro y Febrero) el período activo de pocos juveniles y al 
Tabla 6. Ejemplares de $A$. erythrums vistos durante una jornada completa de cada mes, a tra vés del recorrido asignado. Datos de la temperatura ambiente correspondientes a los días en que se efectuaron los transectos. No figura el sex ratio de 1 as muestras que no son representativas.

Total number of juvenile and adult lizards in a complet journey on different months. Air temperature data corresponds to the work days. The sex ratio of the non re presentative samples does not appear.

\begin{tabular}{|c|rrr|rrr|rr|}
\hline MES & JUV. & ADUL. & M/H & $\mathrm{T}_{7.00}$ & $\mathrm{~T}_{13}$ & $\mathrm{~T}_{18}$ & $\mathrm{~T}_{\text {máx. }}$ & hora \\
\hline ENE & 1 & - & - & 9.6 & 14.0 & 14.0 & 16.0 & 20.00 \\
FEB & 2 & 5 & - & 6.8 & 14.8 & 14.0 & 17.4 & 11.55 \\
MAR & 35 & 27 & $3: 1$ & 8.0 & 14.6 & 12.8 & 15.0 & 14.00 \\
ABR & 46 & 53 & $8: 1$ & 12.8 & 18.0 & 16.2 & 18.5 & 12.10 \\
MAY & 50 & 63 & $5: 2$ & 20.8 & 22.4 & 21.6 & 24.2 & 14.50 \\
JUN & - & 67 & $5: 3$ & 23.6 & 25.6 & 24.6 & 27.6 & 9.15 \\
JUL & 7 & 49 & $1: 1$ & 24.2 & 32.8 & 29.6 & 33.8 & 15.20 \\
AGO & 61 & 50 & $3: 2$ & 22.6 & 28.0 & 26.0 & 30.2 & 15.10 \\
SEP & 72 & 20 & $15: 1$ & 18.0 & 27.0 & 23.8 & 27.0 & 13.00 \\
OCT & 33 & 3 & - & 12.4 & 21.4 & 18.2 & 22.4 & 12.10 \\
NOV & - & 1 & - & 7.4 & 19.4 & 11.4 & 19.7 & 13.30 \\
DIC & 2 & - & - & 6.6 & 16.6 & 14.8 & 18.0 & 14.50 \\
\hline
\end{tabular}



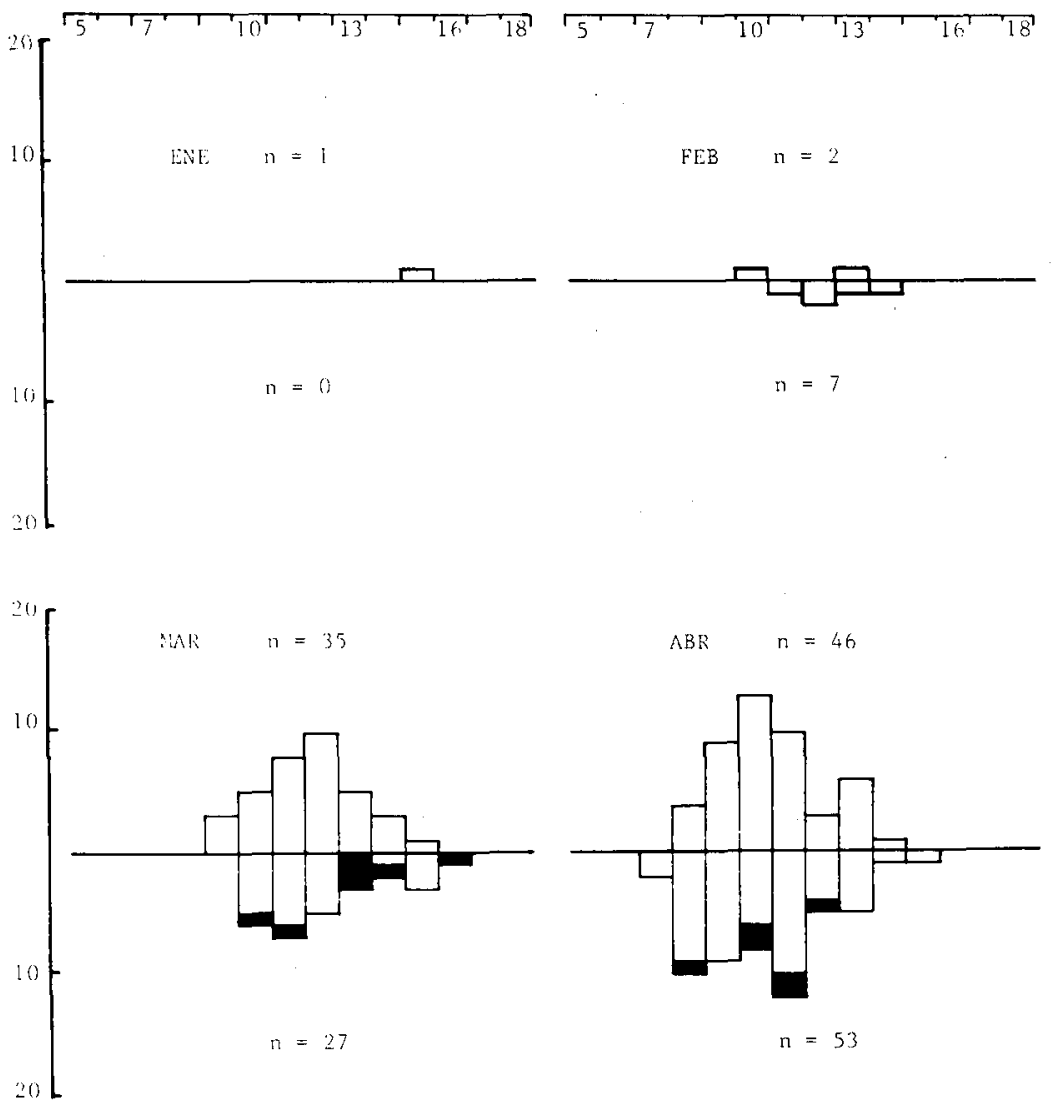

20
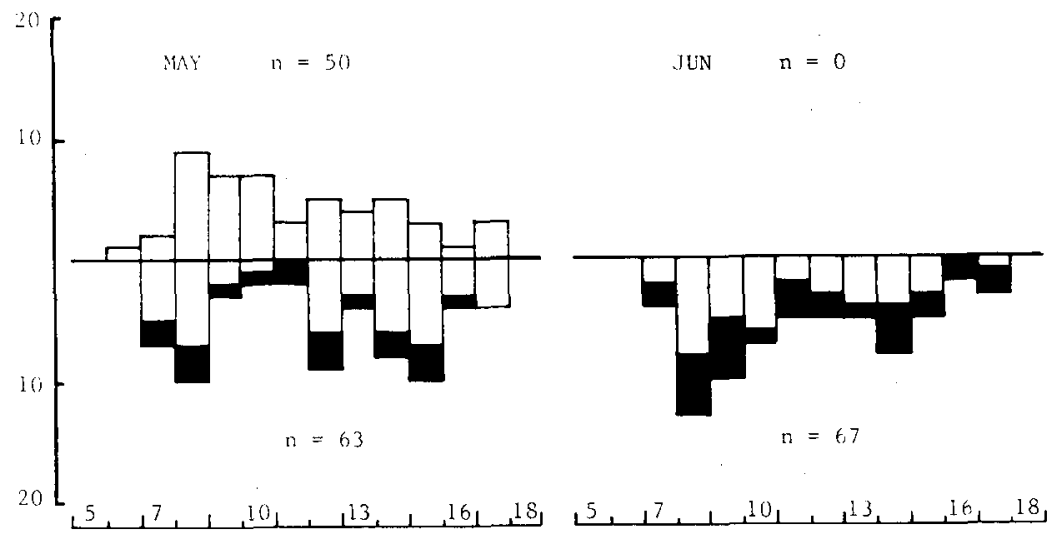

Figura 5 . Histogramas diarios de actividad para los distintos me ses del año para adultos y juveniles de Acanthodactylus erythmums. Se representan en abcisas los intervalos horarios, y en ordenadas el número de ejemplares para ca da intervalo. "n" representa el número total de ejemplares para cada clase de edad. 

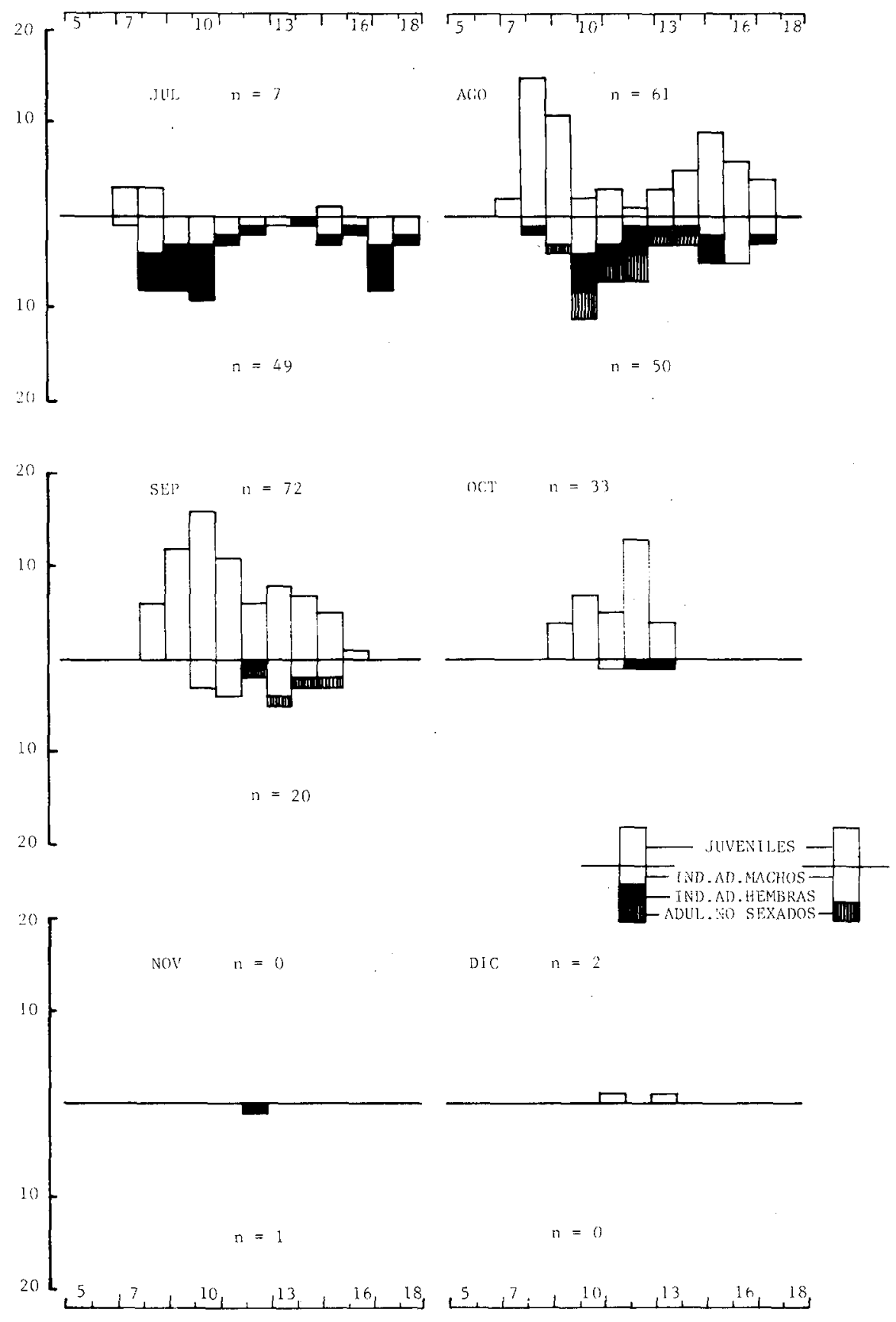

Hourly activity variations in juvenile and adult popula tion of Acanthodactylus erythrurus. Time of day figures in horizontal axis and the number of lizards, in a vertical one. "n" means the population size observed on the transect. 
gún adulto, tiene lugar en las horas de máxima temperatura ambiente.

\section{DISCUSION}

Los reptiles no suelen ocupar el espacio de for ma uniforme en ningunas de las escalas en las que usual mente se estudian (Heatwole, 1976). En realidad no es sencillo encontrar un espacio que se pueda considerar ho mogéneo, ya que existen un gran número de caracteristicas que representan distintos motivos de heterogeneidad que se combinan de maneras casi infinitas y ante las que los organismos presentan opciones de preferencia.

Heatwole (1977) señala que, en la selección del habitat, las especies no recurren $\mathrm{ni}$ a aquellos factores muy fluctuantes, $n i$ a aquellos tan constantes que no per mitan una diferenciación. Es evidente que las especies tienen capacidad de discriminar entre diversas características estructurales del medio y por tanto, seleccionan aquellas combinaciones más favorables. Cuando se pretende este tipo de aproximación autoecológica, la si tuación más favorable se da cuando la especie en cuestión valora también las características elegidas en el estudio.

Para la especie en conjunto, el orden de influen cia de las tres características consideradas, comienzá por la cobertura vegetal, seguida del tipo de vegetación y en último término de la pendiente. La generalización esbozada por Heatwole (1977) de que los reptiles serían más morfólogos que taxónomos o meteorólogos no es 10 contradictoria que puede parecer a primera vista con es tos resultados, ya que la cobertura, y sobre todo el ti po de vegetación, son el reflejo de caracteristicas fí sicas del substrato tales como la granulometría y compa cidad.

Para las diferencias entre sexos y clases de $\underline{e}$ dad, es interesante la inspección de dos tablas resumen que figuran en Schoener (1977), en las que aparecen di ferencias entre distintas dimensiones del nicho, cuyo - 
resultado es la disminución de la competencia, tanto en tre edades (tabla $V$ de las citadas) como entre sexos (tabla VI) . Es señalado como resumen que, la diferencia más citada entre clases de edad, es el tamaño de las pre sas y en segundo lugar, el microhabitat. Entre sexos, por el contrario, se han señalado mayor número de veces diferencias de microhabitat que alimentarias. Al no in cluir el presente estudio datos de alimentación, no re sulta posible ponderar para esta especie el valor relati vo de ambos factores, pero sí es posible afirmar que, de los tres factores del ambiente considerados, la cobertu ra y el tipo de vegetación intervienen en la selección que se lleva a cabo tanto por sexos como.por clases de edad, mientras que la pendiente sólo es "reconocida"por uno de los sexos.

La segregación de zonas de baja cobertura, que va asociada regularmente con el tipo II de vegetación y un substrato de areniscas compactas, parece interpretable tanto desde el punto de vista trófico, al estar bue na parte de la fauna entomológica asociada a los vegeta les de más porte, como de los aspectos que se relacionan con la ubicación de los refugios, en que intervienen tan to la presencia de estos mismos vegetales como la aptitud del substrato para la excavación de las huras.

La preferencia de las hembras, sobre todo las a dultas, por zonas 11 anas, de suelo limoso, gran cobertú ra y vegetación de tipo I, podría venir relacionada tan to con un posible óptimo trófico de estas áreas, a las que acudirian tanto hembras gestantes como post-reproduc toras, como con cierta preferencia por la mayor humedad de estos suelos que facilitarían una mayor viabilidad de las puestas, al disminuir las posibilidades de desecación (Heatwole, 1976).

Debido a que las temperaturas ambiente y de subs trato varían estacionalmente, la actividad temporal de los reptiles está estrechamente ligada a sus requerimien tos termorreguladores (Pianka, 1969; Avery, 1978) . En ambientes como el presente, cuyo substrato está apenas provisto de una leve cubierta vegetal, el rango de tem peraturas ambiente y de substrato es muy amplio, y las temperaturas corporales no han de acompañar obligatoria mente a aquellas, por lo que las pautas de comportamiento termorregulador suelen resultar muy complicadas. 
Parece lógico sugerir que el inicio anticipado de la actividad de los elementos juveniles en la mayoría de los meses, esté relacionado con el mayor valor en la relación sup/vol., lo que facilitaría el intercambio ca lórico con el medio (Heatwole, 1976) y el mínimo voluntario exigible para el inicio de la actividad vendría a delantado en el interior del refugio. Por el mismo moti vo, la hora de retiro al final de la tarde viene antic $\bar{i}$ pada para juveniles en aquellos meses (Marzo y Abril)en los que el descenso de la temperatura ambiente a esa ho ra es brusco.

En especies que no practican enterramiento, es muy posible que el estímulo venga dado por la iluminación directa del emplazamiento del refugio (Saint Girons 1976), aunque Avery (1978) señala para P.muralis y P.si cula la dependencia directa de la temperatura con la hora de salida.

El efecto de la bimodalidad de las curvas es, a simismo, producto de los requerimientos termorreguladores, sensiblemente diferentes en los meses en los que se presenta. Es un fenómeno que viene menguado segün ascen demos en latitud (Avery, 1978 y Saint Girons, 1977), don de las curvas de actividad se tornan unimodales, incluso en los meses de más calor.

Este cese temporal de la actividad, tratando el caso de días completamente despejados, es debido a que el ambiente alcanza una temperatura desfavorablemente al ta para el animal (Heatwole, 1976) incluso a la sombra de grandes arbustos. La completa paralización de la ac tividad no se da en ningún mes en nuestra zona de estú dio como sucede en el trabajo de Busack (1976), en cuyas parcelas la temperatura ambiente ha llegado a superar $\operatorname{los} 40^{\circ} \mathrm{C}$.

Aunque los individuos de esta especie practican la intermitencia de períodos dentro y fuera de los arbus tos, la bimodalidad de las curvas estivales no se debe a un artefacto de este fenómeno. El hecho de que no to dos los ejemplares acudan al refugio en horas de máxima radiación nos indica que este tipo de comportamiento cons tituye un ultimo recurso de ejemplares que han alcanzado una excesiva temperatura corporal . 
Ciertos ejemplares - la mayoría juveniles - perma necen activos en horas de máxima insolación de los meses más fríos, de Octubre a Enero. Esta continuidad anual en la actividad viene condicionada sin duda por la falta de acúmulos en tejidos de reserva, tanto en juveniles como en adultos que permanecen activos, y a la gran re lación sup/vol. de los individuos juveniles (Huey y Pian $\mathrm{ka}, 1977)$.

La respuesta tan inmediata a la máxima temperatura ambiente puede tener relación con la profundidad del refugio elegido y de hecho, Heatwole (1976), señala que el espesor de la capa de substrato que los cubre va ría estacionalmente de acuerdo a la variación de la tem peratura ambiental, por lo que pensamos que las huras de los ejemplares juveniles activos en estos meses se loca lizan a poca profundidad, para responder a las temperaturas máximas del substrato superior en horas de medio día (Busack, 1976). En esas horas, las pautas de compor tamiento han de ser lo suficientemente ajustadas como pá ra alcanzar unas temperaturas corporales que si no son las máximas, han de ser aceptables para las distintas ac tividades .

E1 curioso fenómeno de la disminución progresiva del sex ratio a partir del máximo porcentaje de machos activos en el mes de Abril hasta el de Julio en favor de las hembras adultas, podiamos referenciarlo a la idea de Huey y Pianka (1977) de la falta de acumulación, en ciertas hembras que han realizado gran esfuerzo en las puestas más recientes, de material de reserva, y que es taría acompañado en los meses de Junio y Julio por un in cremento del índice de actividad. Schoener (1977), ba sado en Crews (1974) interpreta esta progresiva desigual dad intersexual en especies estacionales, como el resul tado del restablecimiento anual del territorio por parte de ejemplares machos; en algunas especies, esta abundan te actividad en las hembras vendría según él condiciona da por la necesidad de captación de alimento en períodos gestantes. 


\section{RESUMEN Y CONCLUSIONES}

El presente trabajo describe la distribución es pacial y temporal por sexos y clases de edad de la lagar tija colirroja (Acanthodactylus erythrums, Schinz) en la franja costera de arenal de la Playa del Saladar (aI sur de la ciudad de Alicante).

En la distribución espacial se han considerado características del biotopo, pendiente, y de la biocenosis, cobertura y tipos de vegetación. En el aspecto tem poral, se han anotado cambios diarios y estacionales de la actividad.

Para la especie en conjunto, es clara la preferencia por zonas de poca pendiente, cubiertas en el $10 \%$ o más de su superficie, y en las que aparecen los tipos I y III descritos de vegetación.

Las observaciones por edades y sexos señalan que las hembras - más las adultas que las juveniles - recha zan zonas de mediana y gran pendiente. Respecto a la có bertura, los individuos juveniles machos y adultos hembras, seleccionan más que el resto, las zonas cubiertas más de un $30 \%$ de su superficie. El tipo I de vegetación ob tiene los máximos valores de aceptación por parte de las hembras adultas, con un posible sentido trófico y reproductor . E1 tipo III de vegetación es preferido por hem bras juveniles y en menor grado por adultos machos. E tipo IV es indiferente y el tipo II es segregado en todos los casos.

Excepto en los meses de Abril y Julio, los indi viduos juveniles dan comienzo su actividad diaria antes que los adultos.

Las curvas de actividad son bimodales en los me ses más calurosos, siendo el primer pico mayor que el se gundo, y unimodales para los meses templados como respues ta a dos exigencias distintas en la termorregulación.

Existe continuidad de 1a actividad en los meses más fríos, por parte de algunos elementos (juveniles y,- 
esporádicamente, adultos) y tiene lugar a las horas de máxima temperatura ambiente.

La fracción de hembras adultas frente a la de ma chos aumenta progresivamente en el tiempo comprendido en tre los meses de Abril y Julio.

\section{SUMMARY}

The spatial and temporal distribution by sex and size classes of the fringe-toed lizard (Acanthodactylus erythrurus, Schinz) are described here. This paper is a study of a reptile population in the sand dune ecosystem at EI Saladar beach (Alicante, Spain).

Three factors have been considered in the spatial distribution : slope, cover and different kinds of vegetation. As for the time factor in our observations, we have noted daily and seasonal changes.

The species prefers low slope spots, covered in $10 \%$ and more, and places with types I and III for vegeta tion.

Females (more adults than juveniles) reject me dium and great slope zones. As for cover, male juveniles and adult females select zones covered in $\geq 30 \%$.

Tipo I vegetation is occupied by adult females preferably, because of the trophic and reproductive requirements. Type III is prefered by juvenile females and adult males. Type IV is neutral and type II is always depreciated .

In activity pattern, juvenile individuals begin the daily activity before adult ones, except in April and July .

In summer months, the activity curve appears bi modal because of the thermoregulation exigences, with a first peak greater than the second. In spring and fall 
months, the curve is unimodal .

In winter months the activity continues in a few juveniles when the air temperature is highest in the day.

Sex ratio varies progressively between April and July . 


\section{BIBLIOGRAF IA}

ANONIMO, 1979 . Hoja informativa para R.U.E. (RUExprés) del C.C.P D. del M.E.C. $01: 1-4$.

AVERY, R.A. 1978 . Activity patterns, thermoregulation and food consumption in two sympatric lizard species (Podarcis muralis and P.sicula) from central Italy. Journal of Animal Ecology, 47:143-158.

BONS, N. 1972 . Variations histophysiologiques du tractus genital femelle du lezard Acanthodactylus exythrums Zineomaculatus. Dum \& Bibr. au cours du cycle annuel. Société des Sciences $N a$ turelzes et Physiques du Maroc. Tome 52:59-120.

BUSACK, S.D. 1976 . Activity cycles and body temperatures of Acan thodactylus erythmus. Copeia, 4:826-830.

CISSE, M., KARNS, D.R. y KARNS, K.C. 1977 . Aspects of the ecology of Acanthodactylus dumerizi . Milne-Edwards . (Sauria: Lacertidae) in Senegal . Bul. Inst. Fondam. Afr. Noire Sér. A Sci. Nat. 39(1): 190-218.

CREWS, D. 1974 . Effects of group stability, male-male aggression and male courtship behavior on environmentally induced ovarian recrudescence in the lizard Anolis carolinensis. J.Zool. Lond. 172:419-441.

FLEMING,T.H. y HOOKER, R.S. (1975). Anolis cupreus : The response of a lizard to tropical seasonality. Ecology, 56:1243-1261.

HEATWOLE, H. 1976 . Reptize Ecology . University of Queensland Press. St.Lucia . 178 pp.

HEATWOLE, H. 1977 . Habitat selection in reptiles pp.137-155 en C.Gans y D.W. Tinkle eds. Biology of the Reptilia. Vo1.7. Aca demic Press, New York.

HUEY,R.B. y PIANKA, E.R. 1977 . Seasonal variation in thermoregulatory behavior and body temperature of diurnal Kalahari lizards. Ecology, 58:1066-1075.

PIANKA, E. 1969 . Sympatry of Desert Lizards (Ctenotus) in Western Australia . Ecology, 50:1012-1030.

POUGH, F.H. y BUSACK, S.D. 1978 . Metabolism and Activity of the Spanish fringe-toed lizard (Lacertidae: Acanthodactylus erytiru rus). J.Thermal Biology. Vo1.3:203-205. 
SAINT GIRONS, M.C. 1976 . Relations interéspécifiques et cycle d'activité chez Lacerta viridis et Lacerta agilis (Sauria: La certidae). Vie et Milieu, Vol.XXVI fasc.1 ser.c pp.115-132.

SAINT GIRONS, M.C. 1977 . Le cycle de l'activite chez Lacerta viridis et ses rapports avec la structure sociale. La terre et Za vie. vo1.31:101-116.

SCHOENER, T.W. 1974 . Resource partitioning in ecological communi ties. Science, N.Y. 185: 27-39.

SCHOENER, T.W. 1977 . Competition and the niche . pp. 35-136 en C. Gans and D.W. Tinkle, eds. Biology of the Reptilia. Vol.7, Aca demic Press, New York.

SIMON, C.A. y MIDDENDORF, G.A. 1976 . Resource partitioning by an iguanid lizard : Temporal and microhabitat aspects . Ecology, $57: 1317-1320$.

VALVERDE, J.A. 1967 . Estmuctura de una Comunidad de vertebrados terrestres. I Monografía de la Estación Biológica de Doñana. Consejo Superior de Investigaciones Científicas . Madrid . 